\title{
Measurable Residual Diseases in Haematological Malignancies: Current Applications and Future Direction \\ Salwa Bakr* and Ghada El-Gohary²
}

${ }^{1}$ Department of Clinical Pathology/Hematology, Faculty of Medicine, Fayoum University, Egypt

${ }^{2}$ Department of Clinical Hematology and Bone Marrow Transplantation Unit, Division of Internal Medicine, Ain Shams University, Egypt

\begin{abstract}
Minimal/ measurable residual diseases represents an independent prognostic indicator for several haematological malignancies helping to predict clinical outcome and enables for further assessment of the effectiveness of treatment. The methods of detection of MRD has remarkably improved regarding sensitivity and specificity, and different methods such as real time quantitative polymerase chain reaction (RQ-PCR), multi-parametric flow cytometry (MFC), digital PCR or next generation sequencing (NGS) are currently used in clinical practice. Recently, Digital PCR has been adopted for quantitative assessment of MRD. However, the best method still needs to be determined. While enhancement in standardization of the different MRD approaches was reported, optimal timing and specific threshold for intervention need to be defined. Therefore well-designed clinical studies are required to diminish the risk of relapse and improve overall survival.
\end{abstract}

\section{Keywords: Minimal residual diseases; MFC; RQ-PCR; NGS; dPCR}

\section{Introduction}

Minimal residual disease (MRD) or the persistence of occult submicroscopic leukemic cells after initial phase of induction chemotherapy, is a powerful tool in predicting relapse in hematologic malignancy [1].

Several studies have demonstrated that quantitative assessment of MRD or more precisely termed measurable residual diseases represents an independent prognostic indicator for several haematological malignancies helping to predict patient's clinical outcome and enables for further assessment of the effectiveness of treatment regimen [2-6]. The most important seems also individualization of treatment based on analysis of the results of MRD [5]. However, the clinical impact of MRD detection in the different haematologic malignancies is varied. In Acute leukaemia, the main clinical role of MRD evaluation is the assessment of early treatment response. While, in chronic myeloid leukaemia (CML) and acute promyelocytic leukaemia (APL), monitoring of MRD levels over a period of time necessitates clinically for early detection of patients at high risk of relapse as well as for adjusting treatment. Similarly, this might be applied in mature lymphoid malignancy. Furthermore, the clinical application of MRD can be useful for several other specific aims, such as improved staging of lymphomas, early diagnosis of lymphoma and leukaemia in patients with unexplained cytopenias, residual minimal involvement of central nervous system in ALL, and the early detection of malignant cells in autologous stem-cell grafts. However, further studies are required to fully define clinically relevant MRD specific of different hematological malignancies 'MRD windows' (time-span and minimal sensitivity) [7].

Since the major role of MRD rely on the recognition of malignant/ leukaemic cells among normal cells, the main goal to assess level of MRD is by using the most reliable techniques which have, in addition to the high sensitivity of at least 103, specificity, easy standardisation, reproducibility between laboratories, quantifying MRD and rapid collection of results [7].

There are various approaches applicable for detection of MRD which differ in sensitivity and specificity of used leukemic markers [8]. These include morphologic analysis (cytological, cytochemical, and histological), conventional cytogenetics, fluorescent in situ hybridization (FISH), Immunophenotypic analysis (IP), and molecular genetics based techniques [6,7,9-11]. Many of these approaches are not suitable for clinical assessment of MRD because of limited specificity, sensitivity, or applicability [7]. For instance, morphologic assessment, even with the aid of immunohistochemical testing, lacks both specificity and sensitivity. Likewise, conventional cytogenetics and FISH lack the necessary needed sensitivity to detect sub-microscopic leukemic cells [12].

Therefore, several new challenging techniques characterised by increased sensitivity have recently been developed for detection of MRD levels which enables reliable evaluation of the clinical outcome [8]. These techniques offer the sensitivity of one neoplastic cell per $10^{4}$ $10^{6}$ normal cells, which makes them $10^{2}-10^{4}$ times more sensitive than cytomorphologic techniques and are sufficiently specific, quantitative and broadly applicable [13].

At present, flow-cytometery and polymerase chain reaction (PCR) based molecular methods are the main methods applied widely to assess MRD in various setting [12].

With the aim to improve the sensitivity of the traditional PCR techniques, nested PCR, reverse transcriptase-PCR (RT-PCR), nested RT-PCR have been recommended to accurate assessment of MRD with a high sensitivity of $10^{-5}-10^{-6}$ [8]. In addition, the European LeukemiaNet (ELN MRD) consensus guidelines for assessing MRD using PCR recommended testing to be performed in triplicates [14].

More recently, Next Generation Sequencing (NGS), Multiparameter Flow Cytometer (MFC), real time quantitative PCR

*Corresponding author: Salwa Bakr, Department of Clinical Pathology/ Hematology, College of Medicine, Fayoum University, P.O Box: 63514, Egypt, Tel: +20 1111034777; E-mail: salwabakr1@hotmail.com

Received October 18, 2019; Accepted November 05, 2019; Published November 09, 2019

Citation: Bakr S, El-Gohary G (2019) Measurable Residual Diseases in Haematological Malignancies: Current Applications and Future Direction. J Blood Lymph 9: 251.

Copyright: (c) 2019 Bakr S, et al. This is an open-access article distributed under the terms of the Creative Commons Attribution License, which permits unrestricted use, distribution, and reproduction in any medium, provided the original author and source are credited. 
(RQ-PCR), and digital PCR have been used for assessing molecular abnormalities in MRD. Measurements of MRD using NGS techniques are under development, but are not ready for routine application outside of clinical trials (Table 1) [15]. Therefore, the current goldstandard measurements of MRD utilize complementary molecular and MFC-based techniques [15].

A challenge associated with molecular approaches is the need for blast DNA to define a malignant clone, which might be unsuccessful in some cases as well as it is labor intensive. PCR techniques can be successfully applied to identify specific genetic alteration such as chimeric fusion-genes of chromosome aberrations, or the unique gene rearrangements of immunoglobulin (IGH) or T-cell receptor (TCR), and are highly sensitive [10]. However, the ongoing and secondary clonal rearrangements and the phenotypic switch in some disease categories, poses a risk of false-negative results [7]. Hence, MFC has been developed to overcome this issue. While MFC is superior to molecular in terms of rapidity and cost, it requires consistency in approach [16].

Each technique for detecting MRD has pros and cons, which have to be weighted up carefully before making an appropriate choice for each disease [7]. In AML, for instance, molecular MRD assessment should be limited to APL, core-binding factor (CBF) and NPM1, while MFC MRD assessment should be used for other patients who are not molecularly defined. This recommendation may change over time with emerging data for other molecular subgroups [15].

Currently, there is no international consensus for the best method of measuring MRD. Therefore, efforts should be taken much more to define the respective and best technique to use for MRD of each hematological malignancies to improve patients care and also to reduce costs in hematology in the next years [11].

In addition to the difference in sensitivity of each MRD methodology, it differs in the proportion of patients to whom it can be applied. Therefore, sufficient standardization of laboratory based strategies of these MRD assays is one of the challenges nowadays [15].

Furthermore, the application of the standardized protocols (how and when to measure MRD) in routine clinical practice of several haematological malignancies still need a lot of efforts for its broad application [11], in spite of the recently published comprehensive guidelines by the ELN $[1,11]$

\section{Current State of the Art in Detection of MRD}

There are two different approaches have been used to assess MRD. "Leukemia-Associated Immunophenotype (LAIP)" approach relies on the detection and knowledge of the respective character of the leukemic cells at diagnosis, while the "Different-from-Normal (DfN)" approach relies on the knowledge of normal bone marrow and differences from this detected at the time of MRD assessment. LAIP approach enables a more reliable definition at follow-up of aberrancies already present at diagnosis (identifies immunophenotypic shifts), while the DfN approach measures aberrancies present at follow-ups [11].

The final recommendation on MRD assessment by MRD working party of ELN (ELN MRD) during 2016-2017 were subdivided into three categories including MFC MRD, molecular MRD and clinical MRD [15].

\section{MFC MRD assessment}

MFC technique is very useful for obtaining clinically relevant information in some disease categories [7], however it has not been standardized worldwide [17], and its optimal cutoff is still under debate [16]. Moreover, the antigen switch in blast cells at the time of initial diagnosis in comparison to time of relapse which raises the possibility of false negative results, is another drawback $[16,17]$.

To best define MFC MRD, the ELN MRD Working Party recommends that the two approaches "LAIP" and "DfN" should be combined and termed "LAIP-based DfN approach" [15]. The gold standard approach for detecting MRD termed "DfN" is based on quantitative correlation of normal to abnormal cell surface antigen using standardized antibody panels. The assay uses 16 different cell surface markers in 9 tubes aiming to subtract all normal regenerating cells within the specimen then identification of clusters of abnormal cells within the remaining data set. Assessment of such data necessitates a multidimensional gating approach aiming to maintain the spatial relationships between all parameters. (This is distinctly different from multiparameter flow cytometry which simply refers to collecting multiple characteristics simultaneously). The two antibodies, CD45 and CD34, are added in each tube to provide a means to link data between tubes [18].

In essence of LAIPs based DfN approach, MRD by MFC, in the vast majority of cases and at least for acute leukemias should be based on a minimum of 8 colors (preferably $\geq 8$ colors) [11]. Another prerequisite for tracking of the LAIP-based DfN approach established at diagnosis and emerging aberrancies, is usage the same tubes with the same antibody-fluorochrome combinations at diagnosis and at follow-up aiming for the comparison with the initial diagnosis pattern [15].

Recently with the aim of making MFC MRD the global standard, the EuroFlow consortium developed a test method called Next-Generation Flow MRD (NGF-MRD) to identify and quantify malignant cells using automated software, the EuroFlow database. This method would allow almost complete automation of flow cytometry gating analysis [17]. They also provided standard operating procedures for their panel [19]. A comparative study revealed that NGF exhibited high sensitivity of $10^{-5}-10^{-6}$ in detecting MRD with a good reproducibility in quite parallel to NGS. Studies also demonstrated that automated software results correlate well with those of expert manual analysis. Hence, the NGFMRD method is expected to become widely applied worldwide soon, but its clinical value remains to be demonstrated in future clinical studies [20].

Although most previous studies recommended using $0.1 \%$ as the cut-off threshold to distinguish MRD-positive from MRD-negative cases in most published studies, the ELN MRD Working Party (20162017) suggested for further improvement to perform retrospective analysis for patients with MRD burden $<0.1 \%$ for validation of clinical importance of these very low threshold levels [15].

To harmonize the reporting of MFC MRD, it was recommended

\begin{tabular}{|l|l|l|l|l|l|l|l|l|l|l|l|}
\hline Method (MRD) & Sensitivity & AML & ALL & CML & Myeloma \\
\hline Multi-parameter flow cytometry & $1: 104-1: 106$ & + & + & + & + \\
\hline Molecular approach & $1: 104-1: 106$ & + & + & - \\
\hline
\end{tabular}

Table 1: Methods to detect MRD according to disease entities. 
by the ELN MRD Working Party to acquire between 500,000-1 million events as a minimum number of cells for accurate quantification of MRD burden (excluding all CD45 negative cells and debris) unless the cluster of MRD becomes obvious during acquisition and is recognized by the operator. It was also suggested for further improvement in order to minimize subjectivity in data interpretation in discrimination of LAIPs to use multiparameter displays software analysis program such the Infinicyte ${ }^{\circledR}$ programs or APS system of the Kaluza ${ }^{\circledR}[15]$.

With the increasing number of multicenters embarking MFC MRD studies and the strong needs to improve and optimize the reported data, it is strongly suggested either to establish centralization approach for work-up and analysis or to launch working relationship with experienced centers seeking to design common MRD panels and procedures [15].

\section{Molecular MRD assessment}

The LAIP versus DfN approach is similarly true for the molecular techniques [11]. RQ-PCR which can be used to identify specific gene alterations or chimeric fusion gene [16], was recommended by the ELN MRD Working Party (2016-2017) to be the gold standard approach for MRD assessment for its established high sensitivity [15]. However, newer techniques are still being tested for broad clinical applications such as digital PCR (d-PCR) or next generation sequencing (NGS) [11].

With assessing MRD using RQ-PCR, the ELN MRD Working Party (2016-2017) recommended that the initial sample in which molecular relapse was suspected should be included during the measurement of the repeat sample. In addition, standards should be included that cover the Cycle Threshold (CT) range of the patient samples to ensure linearity of the assay at the measured MRD level. To report molecular MRD results, it was recommended to report absolute copy numbers for RT-PCR results, in addition to the fold increase, to enable the clinician to make his/her own judgment [15].

Digital PCR (dPCR), unlike RQ-PCR which does not need calibration curves, has recently been adopted for quantitative monitoring of MRD. Its principle is based on division of the sample among a large number of reaction wells followed by PCR amplification of the target gene. Interpretation of $\mathrm{dPCR}$ results based on calculating the rate of PCR positive wells to PCR negative ones [17]. Drandi et al. found that $\mathrm{dPCR}$ of immunoglobulin gene rearrangement had reproducibility, sensitivity and accuracy in comparison to RQ-PCR when using peripheral blood and bone marrow of 21 mantle cell lymphoma and 18 multiple myeloma (MM) patients. However, up until now, there have been no data to predict outcomes using dPCR in a controlled clinical setting or multi-laboratory standardization programs [21].

Because of the well-known clonal evolution and the phenotype switch in some disease categories, for instance acute leukaemia, NGS MRD represents a useful approach to assess progression of such diseases. Another advantage of NGS tool is that it can identify the number and DNA sequence of tumor clones within a sample [16]. NGS MRD can, theoretically, be applied to monitor progression of all hematologic malignancy with genetic aberrations [17], as it shows promise to assess MRD with a higher sensitivity of up to 10-6. However, its major drawback is not only the lack international accepted standardization, but the high sequencing error rate which impacts the sensitivity of the method. At present, new error-corrected read technologies, bioinformatics approaches, and analytical techniques may improve the sensitivity [16].
NGS standardization of clinical testing (Nex-StoCT) guidelines that should be considered when launching and validating a clinical NGS workflow, had been published since 2012 by the US Centers for Disease Control and Prevention (CDC). However, NGS MRD still lacks international accepted standardization [22].

With the advancements in NGS to detect low frequency mutations in a heterogeneous population, molecular indexing of an individual template with a unique identifier (UID) before PCR and deep sequencing is promising for detecting rare or ultra-rare mutations. As tagging targeted DNA fragments with UIDs could distinguish true mutations from systemic error of PCR and sequencing methods based on consensus among reads sharing same index, reduction in error rates with improvement in sequencing accuracy can be achieved [23]. Another bioinformatical technique "Digital error suppression of NGS data" using silico elimination of background can also replace the UMI role for correcting the sequencing error [24].

In addition, the recently FDA-approved clonoSEQ assay which uses multiplex PCR and NGS to identify and quantify single VDJ rearrangements, takes advantage of the uniform presence of the gene rearrangement across all cells of an individual tumor to detect MRD in patients with B-cell malignancies including multiple myeloma. Although the method is simple in its perception, IgH locus amplification through multiplex PCR represents a practical challenge due to significant sequence homology in the region and variable primer specificity and kinetics [25].

\section{Clinical application of MRD}

It is foreseeable that the use and the clinical importance of MRD studies will not only lead to more specific, individualized treatment and patient follow up but also reduce toxicity and costs. To refine risk assessment of disease progression/ impending relapse, the ELN MRD Working Party urged to the urgent needs to establish a consensus on the optimal intervals to define MRD to follow up the patients [15]. However, it was suggested that MRD should be assessed prior to consolidation treatment and the post-induction time point closer to consolidation, as well as, at both pre-transplant and post-transplant settings [26].

MRD in AML: The molecular heterogeneity of AML poses substantial challenges to use of MRD as a biomarker [27]. Nevertheless, therapeutic decisions is highly influenced with assessment of MRD [28].

Based on MRD kinetics, leukaemia patients can be stratified into those having high-risk disease requiring more intensive treatment, or having low risk of relapse that might benefit from a reduction in treatment, thus reducing the toxicity [27]. Many studies have proven that patients with early MRD reoccurrence or with persistent MRD can receive salvage chemotherapy and/or proceed to stem cell transplant to avoid future hematologic relapse. Although allogeneic stem cell transplants usually yielding a superior outcome as compared to the chemotherapy alone, it does not abrogate the negative effect of pretransplant MRD or unfavorable genetics. On the other hand, patients without MRD or adverse genetics but with high risk of non-relapse mortality (NRM) can be treated with chemotherapy only with or without autologous transplantation in CR1 [29].

Regarding APL, Thmopson et al. [30] reported that approximately $70 \%$ of patients with PML-RARa-associated APL can be cured using protocols involving all trans-retinoic acid (ATRA) combined with anthracycline-based chemotherapy. Therefore, molecular monitoring 
using RQ-PCR of the PML-RARa rearrangement at 3 monthly intervals has been incorporated into a number of treatment algorithms that may help to identify patients who may require additional therapy rather than patients at low risk who may benefit from sparing unnecessary toxicity.

Today validated molecular MRD targets in AML include the PMLRARA translocation in APL, CBF translocations, and mutations in NPM1 [15]. Yet, there is no consensus on the best method of MRD detection in AML or even the defining thresholds and optimal timing for MRD. Therefore, well designed prospective randomized trials are mandatory [16].

MRD in ALL: In ALL, MRD analysis has emerged as one of the most significant prognostic factors, which is independent of patient age, B-or T-cell origin, or genetic subtype besides its allowance for risk group stratification into different treatment arms, ranging from significant treatment reduction to treatment intensification. It can aid in early recognition of high-risk patients (relapsed disease) who can benefit from receiving bone marrow transplantation and patients with refractory ALL for undergoing novel therapies including CAR T-cell therapy and antibody-based immunotherapies [31].

Blinatumomab (Blincyto), a bispecific CD19 directed CD3 T-cell engager, has shown to be effective in both MRD and relapsed/ refractory B-cell ALL. This exemplifies the use of MRD status to select patients and monitor treatment response. The recent FDA approval expansion of blinatumomab was based on data from the BLAST trial (NCT01207388), which showed that $78 \%$ of adult patients with MRD-positive ALL in hematologic remission after chemotherapy achieved a complete MRD response with blinatumomab and that the MRD response correlated with relapse-free survival and OS. The primary endpoint was complete MRD response status after 1 cycle of blinatumomab. The subsequent FDA approval expansion was the first instance of the use of MRD as a biomarker in a regulatory submission [32].

MRD in CML: Major molecular response (MMR) defined as BCR-ABL (IS) of less than $0.1 \%$ or more than 3-log reduction in BCR/ ABL1 mRNA from the standardized baseline, if RQ-PCR (IS) is not available predicts superior long-term clinical outcomes (PFS/EFS). The achievement of MMR has become a consensus goal of CML therapy. MRD can be used to select and monitor patients who are eligible for treatment discontinuation of tyrosine kinase therapy [33].

Measuring BCR/ABL1 transcripts for monitoring MRD should utilize assays with results based on the International Scale (IS) with the standardized baseline set to 100 percent (molecular response is expressed as $\log$ reduction from 100 percent). Therefore, RQ-PCR assay with a sensitivity of more than 4.5-log reduction from the standardized baseline are recommended for assessing MRD in CML. Furthermore, rising numbers of BCR/ABL1 transcripts post allo-HSCT precede which usually precede haematological and cytogenetic relapse, is used as indicators for intervention with another approaches such as donor lymphocyte infusion (DLI) resulting in long-term disease free survival with molecular remission and RT-PCR negativity. Recent challenge to standardise mRNA quantitation of BCR/ABL using RQ-PCR in a multi-centre study which have employed a lyophilised preparation of a K562 cell line as a potential quality control reagent [34].

\section{MRD in Chronic Lymphocytic Leukemia/Lymphoma (CLL)}

Ladetto et al. [16] reported that both MFC and RQ-PCR are equally suited for detection of MRD in CLL, however, molecular methods are the mainstay for assessing MRD in follicular and mantle cell lymphomas. The absence of identifiable CLL cell line by FCM whether in blood or bone marrow confirms a CR, whereas the re-emergence of MRD in the guise of CD20/CD79b+CD5/CD19 negative cells may serve as an indication for further chemotherapy. In addition, a number of potential prognostic markers (CD38, Zap 70, and somatic hypermutation) are measured using either FISH or MFC to look for residual disease following treatment [35].

Treatment, with the exception of the few patients receiving allo HSCT, was until recently confined to palliation. With the advent of newer therapeutic regimens including the monoclonal antibody Rituximab, cyclophosphamide, and purine analogues (fludarabine), the possibility of complete response (CR) becomes high. More recently, the FDA-approved expansion of the labeling information for venetoclax (Venclexta) with the addition of MRD-negativity data from the MURANO trial (NCT02005471) was also the basis for the approval of venetoclax and rituximab (anti CD 20) as the first chemotherapy-free combination for patients with previously treated CLL. The data showed that among patients with relapsed or refractory CLL, the venetoclaxrituximab combination yielded a significantly higher 2-year PFS rate than the bendamustine-rituximab combination (HR for progression or death, 0.17). MRD status in CLL in this context could be characterized as a treatment response biomarker [35].

\section{MRD in lymphoma}

MRD diagnostics is of high clinical significance in patients with indolent B-cell Non-Hodgkin lymphomas (B-NHL) as it serves as an alternative parameter for evaluating effectiveness of treatment and long-term prognosis. However, the full realization of the potential benefit of MRD in lymphoma care has several important obstacles. One is the lack of incorporation of MRD monitoring in prospective trials evaluating novel treatment strategies. Many ongoing clinical trials in lymphoma do not track MRD as an endpoint [36].

In B-NHL, the most broadly applicable MRD target is the molecular analysis of the junctional regions of the rearranged immunoglobulin heavy-chain gene $(\mathrm{IgH})(\sim 80 \%)$ using RQ-PCR Whereas, chromosomal translocations can be used as MRD surrogate markers in selected lymphoma subtypes such as $t(11 ; 14)$ translocation in MCL and $\mathrm{t}(14 ; 18)$ translocation in FL (Figure 1) [37].

Yet the ability to optimally match treatment modalities to patient is still challenging particularly to do so dynamically through the course of therapy, NGS-MRD were found to have the best potential tool to revolutionize treatment paradigms across all lymphoma subtypes. It might help not only in selecting frontline therapy, but in adapting therapy to response, whether it be with change in treatment, de-escalation or intensification of treatment, or use of maintenance therapies. Recent studies also suggest that it may be able to guide interventions after allogeneic stem cell transplantation [38]. While it was proven that auto stem cell transplant (ASCT) can induce molecular remission in patients with pre-transplant MRD positivity and improves PFS, the expectation that all patients will eventually relapse is still quiet relevant. Hence, MRD monitoring offers the opportunity to initiate anti CD 20 therapy prior to overt relapse [39].

The evolving of new CD79-targeting agent named Polatuzumab vedotin is a humanized anti-CD79b monoclonal antibody conjugated to the auristatin MMAE via a cleavable dipeptide valine-citrulline. The CD79 antigen is the signaling component of the B-cell receptor that 
Citation: Bakr S, El-Gohary G (2019) Measurable Residual Diseases in Haematological Malignancies: Current Applications and Future Direction. J Blood Lymph 9: 251.

Page 5 of 7



Figure 1: Molecular Pathways in B-Cell Lymphomagenesis. Illustrates the higher incidence of specific chromosomal translocations of each type of NHL which arise as a clonal transformation of normal B-cell differentiation.

allows for immune response and is expressed on pre-B cells, mature $\mathrm{B}$ cells, and in the majority of NHL and CLL cases. CD79-alpha/CD79beta heterodimers are integral components of the B-cell receptor structure and have a key role in the signaling pathway that regulates cell proliferation, survival, and apoptosis ,this may play a role in the future in treatment of MRD in NHL in the future [40].

The detection of circulating tumor DNA as an innovative tool to assess MRD in lymphomas is still in its infancy [41]. Therefore, it cannot be recommended for routine clinical use yet [42].

Recently, circulating clonal tumor cells of Reed Sternberg cells which is the pathognomonic characteristic for Classical Hodgkin lymphoma $(\mathrm{CHL})$ can be detected in the blood of patients with $\mathrm{CHL}$, providing new opportunities to explore novel methods to detect MRD [43].

Using DNA microarray data analysis for the identification of such HL cell lines for some of those identified genes named SNFT, EBI3, CCL17, and H2AFB can be helpful for further analysis. Moreover, it was found that persistently increased transcript levels of CCL17 and EBI3 were found in blood and were associated with fatal course of the disease [44].

New Era of medications has been evolved that can be used to treat HL with MRD post auto SCT such as; monoclonal antibodies (anti CD 30) named brentuximab vedotin. Besides, the inhibitory signal provided by the programmed cell death 1 (PD-1) that promote immune activation. Those immune checkpoint inhibitors have demonstrated very promising results in both NHL and HL. Therefore, ongoing clinical trials in both NHL and HL are testing the possibility of drug combinations including PD-1 inhibitors with immunomodulating agents and other chemotherapy agents either for salvage, or first line treatment. In addition, further preclinical research is required to dissect the mechanisms via which HL responds to PD-1 blockade [36].

\section{MRD in multiple myeloma (MM)}

Ladetto et al. [16] reported that MRD evaluation of MRD in MM has always been investigated using both molecular and FCM based approaches, unlike other lymphoid neoplasms where molecular methods for MRD detection of IgH or TCR loci clearly represent the "golden standard". As these methodologies are not able to detect extramedullary disease, imaging techniques (e.g., positron emission tomography-computed tomography, magnetic resonance imaging) are essential in combination with MRD to assess response [45].

\section{Conclusion}

The methods of detection of MRD has remarkably improved regarding sensitivity and specificity, and different methods such as RQ-PCR, MFC, dPCR or NGS are currently used in clinical practice. However, the best method still needs to be determined. MFC is a crucial clinical tool for detecting MRD particularly after the recently developed protocol of the EuroFlow consortium which attains detection with reproducibly high sensitivity $\left(10^{-5}-10^{-6}\right)$. Recently, dPCR has been adopted for quantitative assessment of MRD. It is superior to RQ-PCR in term of simplicity, as it does not require calibration curve. Although it is cheaper than NGS, it cannot detect MRD when new mutations occur. NGS represents a useful tool to monitor MRD in some disease categories however its current major drawback is the lack of international accepted standardization. While enhancement in standardization of the different MRD approaches was reported, optimal timing and defining thresholds for each need to be evaluated. Therefore well-designed clinical studies are required to diminish the risk of relapse and improve overall survival.

\section{Conflict of Interest}

The author declares no conflict of interest. Ghada EIGohary critical writing and approval of the final submitted version. 
Citation: Bakr S, El-Gohary G (2019) Measurable Residual Diseases in Haematological Malignancies: Current Applications and Future Direction. J Blood Lymph 9: 251.

\section{References}

1. Dunbar A, Tallman M (2018) Molecular Minimal Residual Disease Detection Shows Further Promise in Acute Myeloid Leukemia. The ASCO Post.

2. Dibenedetto SP, Nigro LL, Mayer SP, Rovera G, Schilirò G (1997) Detectable molecular residual disease at the beginning of maintenance therapy indicates poor outcome in children with T-cell acute lymphoblastic leukemia. Blood 90: 1226-1232.

3. Campana D, Coustan-Smith E (1999) Detection of minimal residual disease in acute leukemia by flow cytometry. Cytometry 38: 139-152.

4. Malec M, van der Velden VH, Bjorklund E, Wijkhuijs JM, Soderhall S, et al (2004) Analysis of minimal residual disease in childhood acute lymphoblastic leukemia: comparison between RQ-PCR analysis of $\mathrm{lg} / \mathrm{TcR}$ gene rearrangements and multicolor flow cytometric immunophenotyping. Leukemia 18: $1630-1636$.

5. Willemse MJ, Seriu T, Hettinger K, d'Aniello E, Hop WCJ, et al. (2002) Detection of minimal residual disease identifies differences in treatment response between T-ALL and precursor B-ALL. Blood 99: 4386-4393.

6. Bader P, Hancock J, Kreyenberg H, Goulden NJ, Niethammer D, et al (2002) Minimal residual disease (MRD) status prior to allogeneic stem cell transplantation is a powerful predictor for post-transplant outcome in children with ALL. Leukemia 16: 1668-1672.

7. Szczepariski T, Orfão A, van der Valden VH, San Miguel JF, van Dongen JJ (2001) Minimal residual disease in leukaemia patients. The lancet oncology 2 : 409-417.

8. Jółkowska J, Derwich K, Dawidowska M (2007) Methods of minimal residual disease (MRD) detection in childhood haematological malignancies. Journal of applied genetics 48: 77-83

9. Dölken, G (2001) Detection of minimal residual disease. Advance in Cancer Research 82: 133-185.

10. Trent R (2005) Complex Genetic Traits. Molecular Medicine (3rdedn) 77-118.

11. Haferlach $T$ (2008) Methods to detect minimal residual disease. HemaSphere 2: $160-161$.

12. Wake L, Chen X (2019) Minimal Residual Disease Testing in Acute Lymphoblastic Leukemia/Lymphoma. Minimal Residual Disease Testing Current Innovations and Future Direction. Switzerland, Spring International Publishing AG, Part of Spring Nature 23-69

13. Zwick D, Cooley L, Hetherington M (2006) Minimal residual disease testing of acute leukemia by flow cytometry immunophenotyping: a retrospective comparison of detection rates with flow cytometry DNA ploidy or FISH-based methods. Lab Hematol 12: 75-81.

14. Zehentner B (2019) Molecular Diagnostics for Minimal Residual Disease Analysis in Hematopoietic Malignancies. Minimal Residual Disease Testing Current Innovations and Future Direction. Switzerland, Spring International Publishing AG, Part of Spring, Nature pp: 69-101.

15. Schuurhuis GJ, Heuser M, Freeman S, Béné MC, Buccisano F, et al. (2018) Minimal/measurable residual disease in AML: a consensus document from the European LeukemiaNet MRD Working Party. Blood 131: 1275-1291.

16. Ladetto M, Böttcher S, Kröger N, Pulsipher MA, Bader P (2018) Methods and role of minimal residual disease after stem cell transplantation. Bone marrow transplantation 54: 681-690.

17. Takamatsu H (2017) Comparison of Minimal Residual Disease Detection by Multiparameter Flow Cytometry, ASO-qPCR, Droplet Digital PCR, and Deep Sequencing in Patients with Multiple Myeloma Who Underwent Autologous Stem Cell Transplantation. J Clin Med 6: 91

18. Loken M, Brodersen L, Wells D (2019) Monitoring AML Response Using "Difference from Normal" Flow Cytometry. Minimal Residual Disease Testing Current Innovations and Future Direction. Switzerland, Spring International Publishing AG, Part of Spring Nature P: 101-139.

19. Kalina T, Flores-Montero J, van der Velden VHJ, Martin-Ayuso M, Böttcher S, et al. (2012) EuroFlow standardization offlow cytometer instrument settings and immunophenotyping protocols. Leukemia 26: 1986-2010.

20. Flores-Montero J, Sanoja-Flores L, Paiva B, Puig N, Garcia-Sanchez O, et al (2017) Next Generation Flow for highly sensitive and standardized detection of minimal residual disease in multiple myeloma. Leukemia 31: 2094-2103.
21. Drandi D, Kubiczkova-Besse L, Ferrero S, Dani N, Passera R, et al. (2015) Minimal Residual Disease Detection by Droplet Digital PCR in Multiple Myeloma, Mantle Cell Lymphoma, and Follicular Lymphoma: A Comparison with Real-Time PCR. J Mol Diagn 17: 652-660.

22. Crowgey EL, Mahajan N (2019) Advancement in Next Generation Sequencing for Detecting Minimal Residual Disease. Minimal Residual Disease Testing Current Innovations and Future Direction. Switzerland, Spring International Publishing AG, Part of Spring Nature pp: 159-193.

23. Kou R, Lam H, Duan H, Ye L, Jongkam N, et al. (2016) Benefits and challenges with applying unique molecular identifiers in next generation sequencing to detect low frequency mutations. PLoS One 11: e0146638.

24. Chen X, Wood BL (2017) Monitoring minimal residual disease in acute leukemia: technical challenges and interpretive complexities. Blood Rev 31 : $63-75$

25. Pistofidis RS, Dong S, Ghobrial IM (2018) In Search of Missed Tumors: NextGeneration Sequencing for Minimal Residual Disease Detection in Multiple Myeloma Comes of Age. The Hematologist ASH news and reports 16.

26. Umukoro C (2018) MRD assessment in AML - Recommendations from the ELN MRD Working Party

27. Brennan Z (2018) Will Blincyto's expanded FDA approval open the door fo wider use of MRD as a biomarker or endpoint? Regulatory Affairs Professional Society website.

28. Ivey A, Hills RK, Simpson MA, Jovanovic JV, Gilkes A, et al. (2016) Assessment of minimal residual disease in standard-risk AML. N Engl J Med 374: 422-433.

29. Döhner H, Estey E, Grimwade D, Amadori S, Appelbaum FR, et al. (2017) Diagnosis and management of AML in adults: 2017 ELN recommendations from an international expert panel. Blood 129: 424-447.

30. Thompson M, Brander D, Nabhan C, Mato A (2018) Minimal residual disease in chronic lymphocytic leukemia in the era of novel agents: a review. JAMA oncol 4: 394-400.

31. Raetz EA, Teachey DT (2016) T-cell acute lymphoblastic leukemia. Hematology Am Soc Hematol Educ Program 2016: 580-588.

32. Gökbuget N, Dombret H, Bonifacio M, Reichle A, Graux C, et al. (2018) Blinatumomab for minimal residual disease in adults with B-cell precursor acute lymphoblastic leukemia. Blood 131: 1522-1531.

33. Marum JE, Branford S (2016) Current developments in molecular monitoring in chronic myeloid leukemia. Ther Adv Hematol 7: 237-251.

34. Cross N, White H, Colomer D, Ehrencrona H, Foroni L, et al. (2015) Laboratory recommendations for scoring deep molecular responses following treatment for chronic myeloid leukemia. Leukemia 29: 999-1003.

35. Seymour JF, Kipps TJ, Eichhorst B, Hillmen P, D'Rozario J, et al. (2018) Venetoclax-rituximab in relapsed or refractory chronic lymphocytic leukemia. N Engl J Med 378: 1107-1120.

36. Anagnostou T, Ansell SM (2017) Responses and response evaluation of immune checkpoint inhibitors in lymphoma. Annals of Lymphoma 1: 1-6.

37. Pott $C$, Brüggemann $M$, Ritgen $M$, van der Velden VH, van Dongen JJ, et al. (2013) MRD detection in B-cell non-Hodgkin lymphomas using Ig gene rearrangements and chromosomal translocations as targets for real-time quantitative PCR. Methods Mol Biol 971: 175-200.

38. Chase ML, Armand $P$ (2018) Minimal residual disease in non-Hodgkin lymphoma - current applications and future directions. Br J Haematol 180: 177-188.

39. Kolstad A, Pedersen LB, Eskelund CW, Husby S, Grønbæk K, et al. (2016) Molecular monitoring and tailored strategy with pre-emptive rituximab treatment for molecular relapse; results from the nordic mantle cell lymphoma studies (MCL2 and MCL3) with median followup of 8.5 years. Blood 128: 146.

40. Hoffmann RM, Coumbe BG, Josephs DH, Mele S, llieva KM, et al. (2018) Antibody structure and engineering considerations for the design and function of Antibody Drug Conjugates (ADCs). Oncoimmunology 7: e1395127.

41. Herrera AF, Kim HT, Kong KA, Faham M, Sun H, et al. (2016) Next- generation sequencing-based detection of circulating tumour DNA After allogeneic stem cell transplantation for lymphoma. Br J Haematol 175: 841-850.

42. Algrin C, Golmard JL, Michallet M, Reman O, Huynh A, et al. (2017) Flow cytometry minimal residual disease after allogeneic transplant for chronic lymphocytic leukemia. Eur J Haematol 98: 363-370. 
Citation: Bakr S, El-Gohary G (2019) Measurable Residual Diseases in Haematological Malignancies: Current Applications and Future Direction. J Blood Lymph 9: 251.

Page 7 of 7

43. Neelapu SS, Fanale MA, Kwak LW, Fayad LE, Rodriguez A, et al. (2013) Detection of classical hodgkin lymphoma in peripheral blood using highthroughput sequencing assay. Blood 122: 627

44. Staege MS, Volkmer I, Kewitz S, Körholz D (2014) Hodgkin's Lymphoma
Specific Transcripts and Minimal Residual Disease Detection. Klinische Pädiatrie 226: 17.

45. Paiva B, García-Sanz R, San Miguel JF (2016) Multiple Myeloma Minima Residual Disease Cancer Treat Res 169: 103-122. 